\title{
Influence of the excitation area on the thresholds of organic second-order distributed feedback lasers
}

\author{
Eva M. Calzado, ${ }^{1}$ José M. Villalvilla, ${ }^{2}$ Pedro G. Boj, ${ }^{3}$ José A. Quintana, ${ }^{3}$ \\ Victor Navarro-Fuster, ${ }^{2}$ Aritz Retolaza, ${ }^{4}$ Santos Merino,${ }^{4}$ and María A. Díaz-García 2 a) \\ ${ }^{1}$ Dpto. de Física, Ingeniería de Sistemas y Teoría de la señal and Instituto Universitario de Materiales de \\ Alicante (IUMA), Universidad de Alicante, Alicante 03080, Spain \\ ${ }^{2}$ Dpto. de Física Aplicada and IUMA, Universidad de Alicante, Alicante 03080, Spain \\ ${ }^{3}$ Dpto. de Óptica and IUMA, Universidad de Alicante, Alicante 03080, Spain \\ ${ }^{4}$ Micro and Nanotechnology Department; IK4-Tekniker, Eibar 20600, Spain
}

(Received 10 July 2012; accepted 5 November 2012; published online 27 November 2012)

\begin{abstract}
It is shown that the optical pump power (or energy) density thresholds required to obtain lasing from organic second-order distributed feedback lasers, increase when the excitation area $(A)$ is smaller than a certain value $\left(A_{\text {crit }}\right)$. So, in order to obtain the minimum possible thresholds and to ensure that they constitute adequate quantities for comparison purposes, the condition $A>A_{\text {crit }}$ should be fulfilled. Results also indicate that when $A<A_{\text {crit }}\left(A_{\text {crit }} \sim 0.1 \mathrm{~mm}^{2}\right.$ for the devices studied here), the operational device lifetime, which depends mainly on the pump power (or energy) density, becomes drastically reduced. (C) 2012 American Institute of Physics. [http://dx.doi.org/10.1063/1.4768242]
\end{abstract}

Organic solid-state lasers (OSLs) have been widely investigated due to the advantages of easy processability, chemical versatility, wavelength tuneability, and low cost offered by organic materials. ${ }^{1,2}$ The interest in OSLs increased with the discovery of stimulated emission in optically pumped semiconducting polymer films, ${ }^{3,4}$ since they opened the possibility of using electrical excitation. The goal of obtaining laser diodes was initially the main motivation to decrease the laser thresholds of OSLs, so many works focused in improving the active materials and the resonators. Although diode lasers have not been demonstrated yet, thanks to all these efforts, laser thresholds have been decreased so much that today it is possible to pump with cheap inorganic diode lasers ${ }^{1,5}$ and even with light emitting diodes. ${ }^{6}$ Therefore, these low-cost and compact optically pumped lasers are by themselves useful for applications. ${ }^{1,2}$

Among the various types of OSLs reported in the literature, distributed feedback (DFB) lasers have been particularly successful. ${ }^{1,2}$ So today they are being used to develop applications in the fields of telecommunications, ${ }^{2}$ biosensing, and chemical sensing. ${ }^{7,8}$ In DFB lasers, the active material is deposited as a thin film over an appropriate substrate so it constitutes a waveguide. Feedback is achieved by the incorporation of periodic nanostructures (obtained by modulating either the refractive index or the gain) that Bragg-scatter the light, thus, avoiding the need of good-quality end facets. In a one-dimensional (1D) DFB laser, the wavelength that satisfies the Bragg condition $\left(\lambda_{\mathrm{Bragg}}\right)$ given by

$$
m \cdot \lambda_{\text {Bragg }}=2 \cdot n_{\text {eff }} \cdot \Lambda,
$$

where $m$ is the order of diffraction, $n_{\text {eff }}$ is the effective refractive index of the waveguide, and $\Lambda$ is the grating period, constitutes the resonant wavelength in the cavity, which will then be diffracted in the grating in different directions.

\footnotetext{
${ }^{\text {a) }}$ Author to whom correspondence should be addressed. Electronic mail: maria.diaz@ua.es.
}

For second-order DFBs ( $m=2$ in Eq. (1)), light is coupled out of the film in a direction perpendicular to the waveguide film, by first-order diffraction. DFB resonators can be easily integrated into planar organic waveguides, which is a clear advantage from the fabrication point of view, as compared to other types of laser cavities.

In general, OSLs demand very intense pumping conditions due to the short photoluminescence (PL) lifetimes of the active materials (typically $\leq 1 \mathrm{~ns}$ in the case of fluorescent materials). So, excitation is performed by tightly focusing the pump beam through the gain medium provided by a pulsed laser source. Many of the milestones in achieving low thresholds (expressed as energy per pulse) reported in the literature have implied the use of very small excitation sizes. Thresholds in the $\mathrm{nJ} / \mathrm{pulse}^{5,9,10}$ and even in the $\mathrm{pJ} / \mathrm{pulse}^{11,12}$ regimes have been obtained, with areas typically between 0.03 and $0.1 \mathrm{~mm}^{2}$. From a practical point of view, expressing the threshold as energy per pulse is convenient since one motivation to decrease the threshold (besides the final goal of achieving a laser diode) has been to use more compact excitation sources, which generally provide pulses of lower energy. However, in order to properly assess the threshold performance of a certain device in comparison to others, the threshold should be given as energy density (energy per pulse divided by the area of the excitation beam over the sample), or better as power density (by dividing also by the temporal pulse width). ${ }^{13}$ It should be noted that in order to use these latter units, stationary conditions should apply, which means that the pulse duration should be larger than the PL lifetime. Very often, all these considerations have not been taken into account in the literature, partly due to the already mentioned convenience to use energy per pulse units. As a result, it is often not obvious to establish rankings of devices in terms of threshold. Fortunately, many groups provided their threshold values in various units, being aware that in order to compare their thresholds with those of other devices, power density or energy density units should be used. ${ }^{6,10-14}$ 
In this context, the main objective of this work is to show experimentally that for organic second-order DFB lasers, the pump power (or energy) density thresholds increase considerably when the pump spot size is smaller than a certain value. As a consequence, the assumption that thresholds are comparable whenever they are expressed in power density units is not generally valid. A second aim of this work is to investigate the effect of reducing the excitation area on the laser photostability under ambient conditions, given that this parameter depends strongly on the pump energy (or power) density. ${ }^{15}$

The devices used in this work have been 1D second-order DFB lasers based on polystyrene (PS) films doped with the perylenediimide derivative $\mathrm{N}, \mathrm{N}^{\prime}$-di-(1-hexylheptyl) perylene3,4:9,10-tetracarboxylic diimide (PDI-C6), as active materials. The laser properties of devices based on these materials, with DFB gratings engraved both on the substrate ${ }^{15,16}$ and on the active film, ${ }^{17}$ have already been reported.

DFB gratings with $\Lambda=368 \mathrm{~nm}$ and grating depths $(d)$ of 120 and $400 \mathrm{~nm}$ were engraved on $\mathrm{SiO}_{2}$ films (grown by thermal oxidization of silicon wafers) by using nanoimprint lithography and subsequent etching, as previously described. ${ }^{15,16}$ The gratings were characterized by field emission scanning electron microscopy (FE-SEM, ZEISS Ultra Plus) and atomic force microscopy (AFM, NT-MDT Solver PRO). In all cases, high fidelity pattern transfer was achieved. DFB laser fabrication was completed by spin-coating PS films doped with 0.5 wt. \% of PDI-C6 on top of the $\mathrm{SiO}_{2}$ gratings. For the spincoating process, the percentage of PS with respect to the solvent (toluene) was adjusted in order to obtain film thickness (h) of $600 \mathrm{~nm}$. Film surface did not show corrugations due to the presence of the DFB grating on the substrate (checked with an atomic force microscope). Optical quality was good enough to obtain low-loss waveguide confinement without the need of film polishing after the spin-coating deposition. In a previous publication, a detailed study of the influence of changing film thickness and grating depth on the laser characteristics of DFB devices similar to those prepared here was carried out. ${ }^{16}$ In that work, the emission wavelength was tuned from 554 to $583 \mathrm{~nm}$ by varying $h$ between 240 and $1200 \mathrm{~nm}$. For the particular devices used in the present work (with $h=600 \mathrm{~nm}$ ), DFB emission is associated to the fundamental transverse electric waveguide mode and occurs at around $575 \mathrm{~nm}$. Such film thickness has been chosen because it allows to obtain the lowest thresholds and also because these are independent on the grating depth. ${ }^{16}$

The emission properties of the DFB lasers were investigated under ambient conditions by using a frequencydoubled Nd:YAG laser $(10 \mathrm{~ns}, 10 \mathrm{~Hz})$ operating at $532 \mathrm{~nm}$ as excitation source. The pump laser beam was expanded, collimated, and only the central part was selected in order to ensure uniform intensity. Then, it was focused on the samples by using a spherical lens at a $20^{\circ}$ angle with respect to the normal to the film. The final shape of the excited region was elliptical and its size was varied from 0.008 to $2.9 \mathrm{~mm}^{2}$ by moving the distance between the lens and the sample. The emitted light was collected perpendicularly to the surface with an Ocean Optics USB2000-UV-VIS spectrometer (resolution of $1.3 \mathrm{~nm}$ ) placed at $1 \mathrm{~cm}$ from the sample. Laser thresholds were determined as the pump power densities at which abrupt changes in the slopes of the output intensity versus pump intensity curves were observed. The operational lifetime of the devices was studied by recording the total DFB intensity as a function of time and quantified by means of the photostability halflife $\left(\tau_{1 / 2}\right)$, defined as the time or the number of pump pulses at which the emitted laser intensity decays to half of its maximum value. ${ }^{15}$

Fig. 1 shows the effectiveness of reducing the thresholds, when expressed in energy per pulse units, by reducing the area of the excitation beam over the sample. This reduction is useful, as already discussed, in view of using more compact sources which generally have limitations in the amount of energy per pulse that can deliver. As observed, changing the grating depth does not have any effect on the threshold for the whole range of excitation areas explored. This is in agreement with results obtained previously ${ }^{16}$ with a fixed spot size of $1.2 \mathrm{~mm}^{2}$. The behaviour is clearly linear for data obtained with spot sizes above a certain value, indicating that in those cases threshold normalization by dividing by the excitation area would be valid. On the other hand, the functionality of data obtained with small excitation areas cannot be properly determined from this figure. This can be better analysed by looking at Fig. 2, which represents the thresholds as energy and power density (right and left axes, respectively). As observed, for the range of areas in which a linear behaviour is observed in Fig. 1, a horizontal line is obtained in Fig. 2. Above a certain area, denoted as critical area $\left(A_{\text {crit }}\right)$, which is around $0.1 \mathrm{~mm}^{2}$ for these lasers, the decrease of the energy (or power) density threshold with size is as small as the error in measuring it. Therefore, it can be considered that it is approximately invariant. For a better display of data obtained with small spot sizes and for an easier determination of $A_{\text {crit }}$, a logarithmic scale has been used in the abscissa of Fig. 2. On the contrary, for small excitation areas, energy and power density thresholds depend on the spot size. This indicates that for these cases the behaviour of Fig. 1 is non-linear. The type of dependence observed in Fig. 2 is similar to the one obtained when thresholds are displayed as a function of the length of the excitation spot along the propagation direction $(z)$, i.e., the long axis of the ellipse,

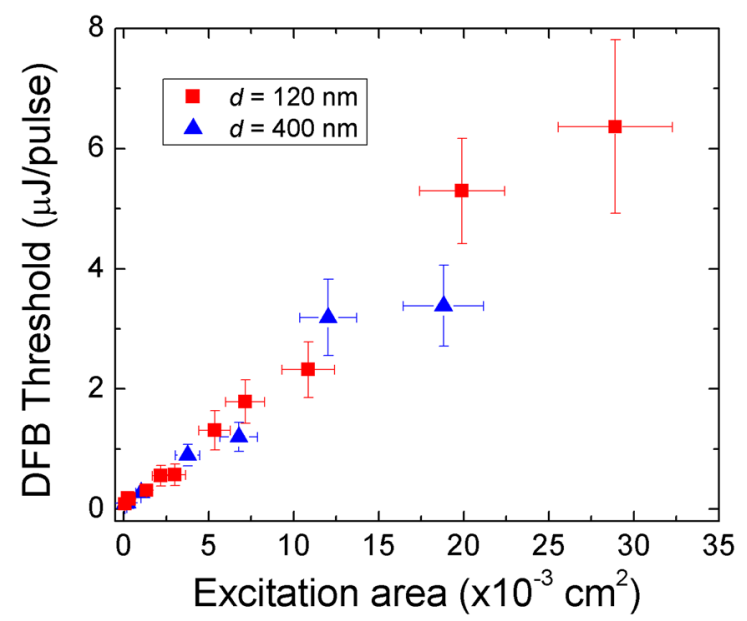

FIG. 1. Laser thresholds, expressed as energy per pulse, for DFB devices with two grating depths, $d$, as a function of the excitation area over the sample. 
(a)

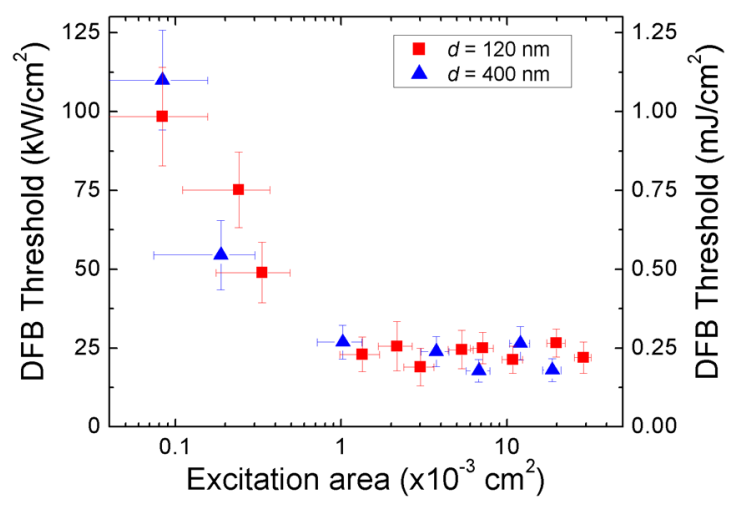

(b)

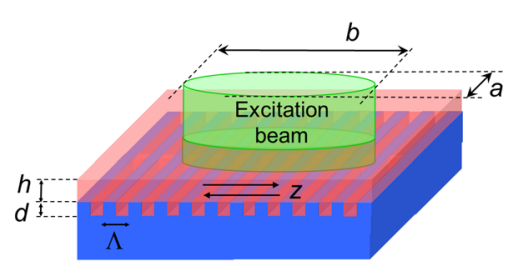

FIG. 2. (a) Laser thresholds, expressed as energy density (right axis) and as power density (left axis), for DFB devices with two grating depths, $d$, as a function of the excitation area; (b) sketch of the excitation geometry.

$b$ (see sketch in Fig. 2(b)). Also in this case, thresholds are independent on $b$ above a certain length, denoted as $b_{\text {crit }}$, which for these devices is approximately $0.37 \mathrm{~mm}$. Below this value, thresholds increase for decreasing $b$. We would like to emphasize the importance of determining experimentally $A_{\text {crit }}$ (or $b_{\text {crit }}$ ) for any DFB laser. First, because this parameter indicates the minimum excitation size needed to obtain the lowest possible threshold (i.e., invariant DFB threshold, $I_{\mathrm{th} \text {-inv }}{ }^{\mathrm{DFB}}$ ); and second, because $I_{\mathrm{th}-\text { inv }}{ }^{\mathrm{DFB}}$ constitutes an adequate quantity for comparison purposes.

The explanation for the threshold decay when the pump size is increased can be obtained from couple wave theory. In the original paper of Kogelnik et al., ${ }^{18}$ it was shown theoretically that for DFB lasers with either pure gain coupling or with either pure index coupling, the gain at threshold decreased when the coupling strength (which is proportional to the index or the gain modulation as well as to the length of the device, $L$ ) increased. For a given device with a certain modulation, the threshold would decrease with increasing $L$, in accordance with results obtained here that show that the threshold decreases when the spot size (and therefore the distance along the propagation direction in which gain is obtained) gets larger. It should be noted that in our case, the existence of a critical length above which the threshold becomes invariant, seems to be better defined than in the theoretical predictions. Various reasons might be responsible for this. First, in Kogelnik's calculations $L$ is the length of the device, while here $b$ is the length of the illuminated area, generally shorter than $L$. In addition, Kogelnik's curves correspond to either pure index coupling or to pure gain coupling, while in our devices both mechanisms contribute to laser emission. ${ }^{16}$ This is in fact a common situation in most DFB lasers reported in the literature (whenever the thickness of the active film varies periodically due to the presence of the DFB structure).
A detailed theoretical analysis of the present problem in order to obtain mathematical expressions for $A_{\text {crit }}$ or $b_{\text {crit }}$ as well as for $I_{\mathrm{th}-\text { inv }}{ }^{\mathrm{DFB}}$ as a function of parameters related to the active material and to the DFB grating structure would be useful. However, the problem is not obvious because in these devices both index and gain coupling are present. In addition, it has been shown experimentally that thresholds depend on other parameters besides the index or the gain modulation. ${ }^{16}$

In previous works dealing with the amplified spontaneous emission (ASE) properties of organic waveguides, the dependence of the ASE threshold intensity with the length of the excitation beam over the sample was investigated. ${ }^{19,20}$ The type of behaviour observed was similar to the one shown by the DFB lasers studied here, i.e., the ASE threshold decreased with increasing excitation length and above a certain critical length, the threshold kept practically invariant. Both quantities, the ASE invariant threshold and the critical length needed to get it, depend on the material's absorption and gain. In particular, the critical length decreases when both material parameters increase. On the other hand, in order to minimize the invariant threshold, high gain and low losses are needed. In a DFB laser, the situation is more complicated, since besides the loss and gain associated to the material, those due to the DFB grating are present. It is well known that in DFB lasers with index modulation, the increase of index contrast $(I C)$ - defined as $\left(n_{\text {eff }}^{\text {high }} / n_{\text {eff }}^{\text {low }}\right)$, where $n_{\text {eff }}^{\text {high }}$ is the effective index of a waveguide of thickness $h+d$ and $n_{\text {eff }}^{\text {low }}$ the one of a waveguide of thickness $h$-leads to higher coupling constants that would increase the gain. ${ }^{16,21}$ However, an increase of $I C$ would also increase the waveguide propagation losses. This latter effect might be significant in very thin films with poor mode confinement, or in devices in which DFB emission appears at higher order modes, for which the maximum of the intensity mode profile is at the interface, where the grating is located. ${ }^{16}$ The problem complicates even further because changing $I C$ also affects the DFB emission wavelength, whose proximity to the maximum of the gain spectrum appears to be the most relevant factor in determining the threshold, as discussed in detail previously. ${ }^{16}$ Consequently, in order to properly analyse the effect of changing $I C$ on $A_{\text {crit }}$ (or $b_{\text {crit }}$ ) and on $I_{\text {th-inv }}{ }_{\text {DFB }}$, devices emitting at similar wavelengths should be considered.

As shown in Fig. 2, the two devices studied in this work (with $d$ values of 120 and $400 \mathrm{~nm}$ and corresponding $I C$ values of 1.006 and 1.014, respectively) have shown similar $A_{\text {crit }}$ and $I_{\text {th-inv }}{ }_{\text {DFB }}$ values. The dependence of $I_{\text {th-inv }}{ }^{\mathrm{DFB}}$ with $I C$ was already studied in Ref. 16 in devices with different $h$ and $d$ values (and therefore different $I C$ values), characterized in all cases with an excitation area well above $A_{\text {crit }}$. The lowest $I_{\text {th-inv }}$ DFB values were obtained for lasers with $h$ between 500 and $1000 \mathrm{~nm}$ (for any $d$ between 30 and $240 \mathrm{~nm}$ ), that emitted close to the maximum of the gain spectrum, which is around $579 \mathrm{~nm}$. In that regime, in which the gain of the material is maximized, thresholds were not affected by changes in $I C$, most likely because the variations induced were too small (of the order of the error in measuring the threshold). The two devices studied in the present work correspond to this regime, since they emit close to the maximum of the gain spectrum, which would justify why 
their $I_{\text {th-inv }}$ DFB values are similar. This explanation could be valid also to justify the similar values obtained for $A_{\text {crit }}$, given that the gain and the loss are the main factors determining $A_{\text {crit }}$. On the other hand, a clear effect on the threshold of changing $I C$ was observed in lasers based on films thinner than $400 \mathrm{~nm} .{ }^{16}$ Those lasers emitted at $\lambda<560 \mathrm{~nm}$ and their thresholds increased considerably with respect to the previous ones, due to the separation from the maximum of the gain spectrum, as well as to a poorer confinement of the waveguide mode. In this regime, the devices with deeper gratings, and therefore, with larger $I C$, showed the highest thresholds. Those results indicated that although the increase of $I C$ leads to a higher gain, the corresponding increase of propagation losses seems to be a dominant factor in this case. Accordingly, it would be expected that for these devices emitting at wavelengths far from the maximum of the gain spectrum, variations in $I C$ would revert on changes on $A_{\text {crit }}$. Additional experiments at this respect, in order to clarify this issue are currently being performed and will be reported elsewhere.

It is remarkable that the excitation areas used in the lasers with the lowest power density thresholds reported to date ${ }^{11,12}$ were below $0.1 \mathrm{~mm}^{2}$, being this value similar to the $A_{\text {crit }}$ found in this work. This does not mean that those thresholds were not properly normalized for comparison purposes, since the $A_{\text {crit }}$ value for those devices might be different than the one obtained here. But our results point out the need to carefully revise results published in the literature by considering dependencies on the excitation area. With respect to the previous works with PDI-based DFB lasers similar to the ones used in the present study, pump spot areas around $1 \mathrm{~mm}^{2}$ were used. ${ }^{15-17}$ This value is above $A_{\text {crit }}$, indicating that the already published thresholds, expressed in energy per pulse units, can be safely normalized to power density units. It should be noted that in our earlier work on organic DFB lasers, thresholds were always expressed in energy per pulse units since we were unsure that threshold normalization could be done, precisely due to the observation that thresholds, although given in energy or power density units, were dependent on the size of the excitation beam over the sample. Some preliminary discussions at this respect can be found elsewhere. ${ }^{17,22}$

We also investigated the effect of changing the size of the excitation beam on the operational lifetime of the devices. As expected, when excitation was performed with spot areas larger than $A_{\text {crit }}$, so the energy and power density thresholds were the same, similar lifetimes (considering that the experimental errors is around $10 \%$ ) were obtained. For example, for the device with $d=400 \mathrm{~nm}, \tau_{1 / 2}$ values of 275 and $330 \mathrm{~min}$ (i.e., $168 \times 10^{3}$ and $198 \times 10^{3}$ pump pulses), for spot sizes of $3.5 \times 10^{-3}$ and $12 \times 10^{-3} \mathrm{~cm}^{2}$, respectively, were measured. Excitation was performed at $50 \mathrm{~kW} / \mathrm{cm}^{2}$ (around two times above the power density threshold, which is the same in both cases). On the other hand, when very small areas (below $A_{\text {crit }}$ ) were used, lifetimes became drastically reduced. This is illustrated in Fig. 3 which shows the evolution of the laser intensity over the time and over the number of pump pulses for a device excited with two different excitation areas, one above and one below $A_{\text {crit }}$, at pump intensities two times above their corresponding thresholds. A

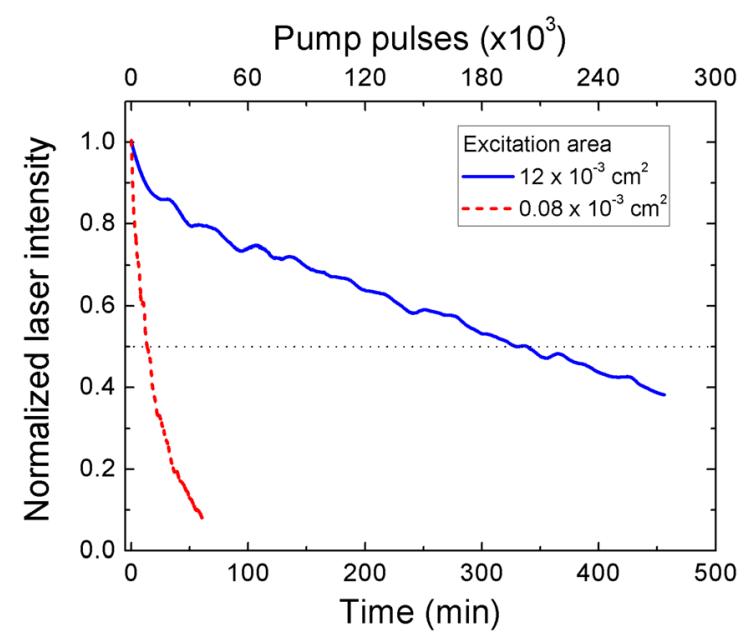

FIG. 3. Normalized laser intensity versus irradiation time (bottom axis) and versus the number of pump pulses $(10 \mathrm{~ns}, 10 \mathrm{~Hz}$; top axis) for a DFB device with $d=400 \mathrm{~nm}$, under excitation with areas below and above $A_{\text {crit }}$ $\sim 1 \times 10^{-3} \mathrm{~cm}^{2}$, at pump power densities two times above the corresponding thresholds (215 and $47 \mathrm{~kW} / \mathrm{cm}^{2}$, respectively).

$\tau_{1 / 2}$ value of only $15 \mathrm{~min}$ (i.e., $9 \times 10^{3}$ pump pulses) was obtained for an area of $0.08 \times 10^{-3} \mathrm{~cm}^{2}$, which implies a reduction in around 20 times with respect to the value obtained with a larger area. The fact that devices degrade faster when the excitation area is below $A_{\text {crit }}$ is not a direct consequence of the size, but to the fact that the pump energy (or power) density needed to obtain laser emission is larger, given that threshold is higher. As shown in Fig. 2(a), the power density threshold increases considerably when the pump size is reduced. So in order to excite in conditions similar to the ones used with the bigger size (two times above threshold), much larger power densities are needed. This result indicates that although from the point of view of threshold, the reduction of the pumped area might be convenient since it allows using excitation sources with pulses of smaller energies, the operational lifetime of the devices decreases drastically. This trade-off between low energy per pulse threshold (if achieved by means of reducing the pump size) and operational durability limits the simultaneous optimization of both parameters when designing a given DFB device.

Concerning the mechanisms responsible for the photodegradation of PDI-doped polymer films, they have been studied by several groups. ${ }^{23,24}$ Two dominant mechanisms have been proposed: type II photooxidation and partially reversible photoreduction, prevailing under aerobic and anaerobic conditions, respectively. For other materials, such as semiconducting polymers, the presence of triplet states seems to play a major role in their photodegradation..$^{25}$ The results obtained in the present work indicate that, despite the type of mechanism dominating the photodegradation, it seems to be independent on the size (at least for the range of values investigated). So, it appears that there are no specific degradation processes linked to the fact that the spot size becomes very small. In accordance with the previous studies on DFB lasers based of PDI-doped films, ${ }^{15,17}$ the parameter affecting more drastically the laser photostability is the pump energy (or power) density.

In conclusion, we have shown that the pump power (or energy) density thresholds of organic second-order DFB 
lasers increase when the pump spot area is smaller than a certain value, denoted as critical area $\left(A_{\text {crit }}\right)$, which is around $0.1 \mathrm{~mm}^{2}$ for the devices studied in this work. This result has three important consequences: (1) Thresholds cannot always be compared, despite they are given as power density; (2) in order to operate in optimized conditions, lasers should be pumped with excitation areas sufficiently large $\left(>A_{\text {crit }}\right)$, which establish limitations in the overall size of the devices; and (3) the laser operational lifetime (i.e., the photostability), which depends strongly on the pump energy (or power) density, gets drastically reduced when very small excitation areas $\left(<A_{\text {crit }}\right)$ are used.

We thank support from the Spanish Government (MECC) and the European Community (FEDER) through Grant Nos. MAT2008-06648-C02 and MAT2011-28167-C02 and to the University of Alicante through Grant No. GRE10-07.

${ }^{1}$ I. D. W. Samuel and G. A. Turnbull, Chem. Rev. 107, 1272 (2007).

${ }^{2}$ J. Clark and G. Lanzani, Nat. Photonics 4, 438 (2010).

${ }^{3}$ F. Hide, M. A. Diaz-Garcia, B. J. Schwartz, M. R. Andersson, Q. B. Pei, and A. J. Heeger, Science 273, 1833 (1996).

${ }^{4}$ N. Tessler, G. J. Denton, and R. H. Friend, Nature 382, 695 (1996).

${ }^{5}$ G. Heliotis, R. Xia, D. D. C. Bradley, G. A. Turnbull, I. D. W. Samuel, P. Andrew, and W. L. Barnes, Appl. Phys. Lett. 83, 2118 (2003).

${ }^{6}$ Y. Yang, G. A. Turnbull, and I. D. W. Samuel, Appl. Phys. Lett. 92, 163306 (2008).

${ }^{7}$ M. Lu, S. S. Choi, C. J. Wagner, J. G. Eden, and B. T. Cunningham, Appl. Phys. Lett. 92, 261502 (2008).

${ }^{8}$ M. B. Christiansen, J. M. Lopacinska, M. H. Jakobsen, N. A. Mortensen, M. Dufva, and A. Kristensen, Opt. Express 17, 2722 (2009).

${ }^{9}$ G. Heliotis, S. A. Choulis, G. Itskos, R. Xia, R. Murray, P. N. Stavrinou, and D. D. C. Bradley, Appl. Phys. Lett. 88, 081104 (2006).
${ }^{10}$ G. Heliotis, R. Xia, D. D. C. Bradley, G. A. Turnbull, I. D. W. Samuel, P. Andrew, and W. L. Barnes, J. Appl. Phys. 96, 6959 (2004).

${ }^{11}$ C. Karnutsch, C. Gürtner, V. Haug, U. Lemmer, T. Farrell, B. S. Nehls, U. Scherf, J. Wang, T. Weimann, G. Heliotis, C. Pflumm, J. C. deMello, and D. D. C. Bradley, Appl. Phys. Lett. 89, 201108 (2006).

${ }^{12}$ R. Xia, W.-Y. Lai, P. A. Levermore, W. Huang, and D. D. C. Bradley, Adv. Funct. Mater. 19, 2844 (2009).

${ }^{13}$ E. M. Calzado, J. M. Villalvilla, P. G. Boj, J. A. Quintana, R. Gómez, J. L. Segura, and M. A. Díaz-García, J. Phys. Chem. C 111, 13595 (2007).

${ }^{14}$ M. H. Song, D. Kabra, B. Wenger, R. H. Friend, and H. J. Snaith, Adv. Funct. Mater. 19, 2130 (2009).

${ }^{15}$ V. Navarro-Fuster, E. M. Calzado, P. G. Boj, J. A. Quintana, J. M. Villalvilla, M. A. Díaz-García, V. Trabadelo, A. Juarros, A. Retolaza, and S. Merino, Appl. Phys. Lett. 97, 171104 (2010).

${ }^{16}$ V. Navarro-Fuster, I. Vragovic, E. M. Calzado, P. G. Boj, J. A. Quintana, J. M. Villalvilla, A. Retolaza, A. Juarros, D. Otaduy, S. Merino, and M. A. Díaz-García, J. Appl. Phys. 112, 043104 (2012).

${ }^{17}$ M. G. Ramirez, P. G. Boj, V. Navarro-Fuster, I. Vragovic, J. M. Villalvilla, I. Alonso, V. Trabadelo, S. Merino, and M. A. Díaz-García, Opt. Express 19, 22443 (2011).

${ }^{18}$ H. Kogelnik and C. V. Shank, J. Appl. Phys. 43, 2327 (1972).

${ }^{19}$ S. V. Frolov, Z. V. Vardeny, and K. Yoshino, Phys. Rev. B 57, 9141 (1998).

${ }^{20}$ K. N. Bourdakos, L. A. Cury, and A. P. Monkman, Org. Electron. 12, 1142 (2011)

${ }^{21}$ M. M. Jorgensen, S. R. Petersen, M. B. Christiansen, T. Buss, C. L. C. Smith, and A. Kristensen, Appl. Phys. Lett. 96, 231115 (2010).

${ }^{22}$ E. M. Calzado, J. M. Villalvilla, P. G. Boj, J. A. Quintana, P. A. Postigo, and M. A. Díaz-García, Appl. Opt. 49, 463-470 (2010).

${ }^{23}$ N. Tanaka, N. Barashkov, J. Heath, and W. N. Sisk, Appl. Opt. 45, 3846 (2006).

${ }^{24}$ L. Cerdán, A. Costela, G. Durán-Sampedro, I. García-Moreno, M. Calle, M. Juan-y-Seva, J. de Abajo, and G. A. Turnbull, J. Mater. Chem. 22, 8938 (2012)

${ }^{25}$ F. Schindler, J. M. Lupton, J. Feldmann, and U. Scherf, Adv. Mater. 16, 653 (2004). 